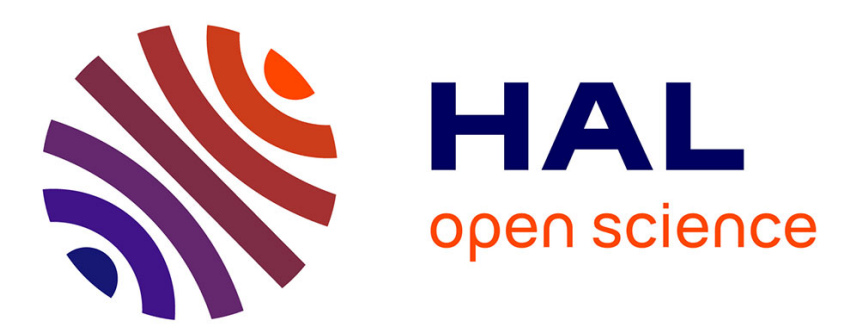

\title{
Strategies using boundary integral equations for solving exterior anisotropic conduction problems outside long cylinders
}

\author{
Alain Corfdir, Guy Bonnet
}

\section{To cite this version:}

Alain Corfdir, Guy Bonnet. Strategies using boundary integral equations for solving exterior anisotropic conduction problems outside long cylinders. Engineering Analysis with Boundary Elements, 2017, 76, pp.1-9. 10.1016/j.enganabound.2016.12.005 . hal-01463881

\section{HAL Id: hal-01463881 \\ https://hal.science/hal-01463881}

Submitted on 9 Feb 2017

HAL is a multi-disciplinary open access archive for the deposit and dissemination of scientific research documents, whether they are published or not. The documents may come from teaching and research institutions in France or abroad, or from public or private research centers.
L'archive ouverte pluridisciplinaire HAL, est destinée au dépôt et à la diffusion de documents scientifiques de niveau recherche, publiés ou non, émanant des établissements d'enseignement et de recherche français ou étrangers, des laboratoires publics ou privés. 


\title{
Strategies using boundary integral equations for solving exterior anisotropic conduction problems outside long cylinders
}

\author{
A. Corfdir ${ }^{\mathrm{a}, *}$, G. Bonnet ${ }^{\mathrm{b}}$ \\ a Laboratoire Navier, UMR 8205, École des Ponts, Ponts, IFSTTAR, UPE, Champs-sur Marne, France \\ ${ }^{\mathrm{b}}$ Université Paris Est, Laboratoire Modélisation et Simulation Multi-Echelle, MSME UMR 8208 CNRS, 5 boulevard Descartes, 77454 Marne la Vallée Cedex, \\ France
}

\section{A R T I C L E I N F O}

\section{Keywords:}

Anisotropy

Boundary integral equation

Plane problems

Green's function

Exterior problem

\begin{abstract}
A B S T R A C T
2D approximations can greatly alleviate the computing effort required to solve anisotropic conduction problems outside long 3D cylindrical domain using boundary integral methods. Two strategies can be used to this aim: either transform the anisotropic conduction problem into an isotropic one, or deal with the anisotropic 2D Green's function. In the first case, it is necessary to provide not only the new features of the transformed domain, but also the new expressions of the boundary conditions over the domain. Conversely, the anisotropic 2D Green's function is defined upto a constant which depends on the length of the cylindrical domain, as shown in the isotropic case. In addition, the use of anisotropic Green's function cannot avoid the occurrence in some cases of degenerate scales, which is well known in the isotropic case. The paper addresses these different points: construction of the anisotropic 2D Green's function and its relation with line sources, description of the transformation leading to an equivalent isotropic problem and finally study of the boundary integral solution of the equivalent $2 \mathrm{D}$ problem, including the occurence of degenerate scales.
\end{abstract}

\section{Introduction}

Conduction problems outside long domains can be solved by using 2D boundary integral equations. However, the consideration of 2D problems involves some difficulties that are known since a long period of time [1] in the isotropic case, i.e. for Laplace equation. The Green's function for the 2D problem can be obtained by integration along a line of the 3D Green's function, a so-called line source (Fig. 1 and Fig. 2) [2]. This primitive is unfortunately infinite not only at the point load but also at infinity. In addition, the integral does not converge for an infinite line source [2]: the integral can be defined only by integration over a finite line source whose length is a priori arbitrary, i.e. a "segment source". Without another information, this length is undetermined and is at least constrained when considering a specific Boundary Value Problem (BVP) solved by Boundary Integral Equation (BIE) for avoiding the occurence of degenerate scale [1], for which the BIE has multiple solutions. Avoiding this inconsistency is possible by restricting the choice of this arbitrary length. However, a case of interest is when the 2D BIE corresponds to the approximation of exterior 3D problems related to a very long cylindrical domain (with an arbitrary section). It has been shown in [3] by different considerations, including slender body expansion [4], for the case of exterior problems of conduction in an isotropic medium that the length used in the 2D Green's function approximates at best the 3D solution near the cylinder when this length is chosen as the height of the cylinder. In this case, the proper 2D Green's function to be used (for the isotropic case) has been shown to be the one computed from a segment source whose length is the one of the cylinder. This paper provides a similar information in the case of anisotropic conduction equation. The problem is described for heat conduction but the same equations can been used for seepage with anisotropic permeability [5], direct electric current with anisotropic resistivity [6], and electrostatics with anisotropic permittivity [7].

The constitutive equations for conduction in an anisotropic medium have been written by several investigators; a comprehensive bibliography for early works can be found in [8]. An important result is that the second order conductivity tensor is symmetrical $[9,10]$ (a recent review can be found in [11]) and that it is always possible to choose the coordinate axes along the eigenvectors, leading to a diagonal conduction tensor.

From another point of view, a suitable change of coordinates can be performed to change a BVP related to anisotropic conduction equations into a BVP with isotropic conduction equations [8,12]. As a result, the Green's functions for anisotropic media can be also obtained by using 


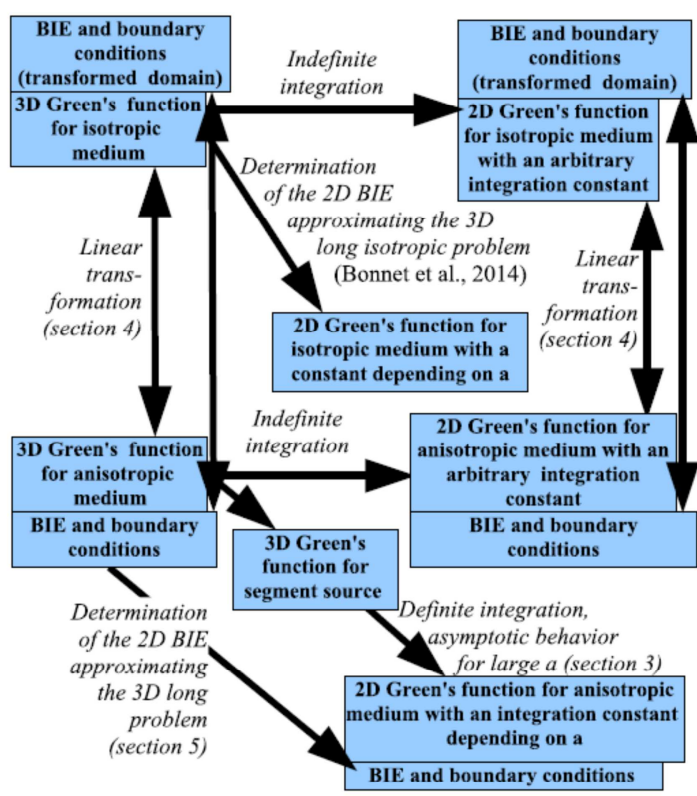

Fig. 1. Relations between the different Green's functions and BIEs: "a" denotes the half length of the cylindrical domain.

an appropriate linear change of coordinates [2,13,14].

Finally, Fig. 1 shows the relations between the different Green's functions related to $2 \mathrm{D}$ or $3 \mathrm{D}$ cases with or without anisotropy. It can be seen that the main problem is the relation between the length of a "long" cylindrical domain and the arbitrary length appearing within the 2D Green's function.

As explained before, the proper Green's function related to a 3D problem over a very long cylindrical domain of length $2 a$ corresponds to a segment source of length $2 a$ for the isotropic case. So, section 2 is devoted to the presentation of Green's functions for anisotropic conduction in 2D and 3D domains. Then, in Section 3, the segment source within a 3D domain is studied, providing a relation between the arbitrary length used in the 2D Green's function and the length of the segment source.

Next, the question of using a convenient characteristic length within the 2D Green's function is treated. In Section 4, it is first recalled how it is possible to reduce the problem for anisotropic conduction to an associated problem for isotropic conduction by using a suitable linear point transformation. Then, the problem of degenerate scale is addressed, and finally, in Section 5, the characteristic length leading to a BIE whose solution approximates at best the 3D solution near the cylindrical boundary is provided.

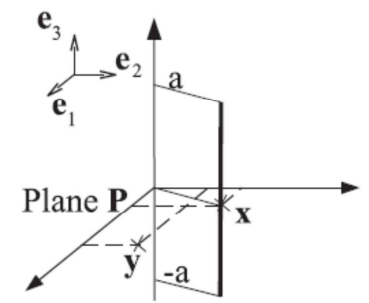

Fig. 2. Geometric notations for a segment source parallel to $\mathbf{e}_{3}$ having its middle (x) in $\mathbf{P}$, the point $\mathbf{y}$ is situated on plane $\mathbf{P}$.

\section{Green's functions for anisotropic conduction equation}

\subsection{Equations for steady-state anisotropic conduction}

The anisotropic conduction equation can be written as the relation between the flux, $\mathbf{q}$, the temperature, $u$, (also named potential) and the anisotropic conductivity tensor $\mathbf{k}$ :

$\mathbf{q}=-\mathbf{k} \cdot \operatorname{grad}(u)$.

If there are no inner sources, the flux conservation leads to:

$\operatorname{div}(\mathbf{q})=\operatorname{div}(\mathbf{k} \cdot \operatorname{grad}(u(\mathbf{y})))=\operatorname{div}\left(\mathbf{k} \cdot \frac{\partial u}{\partial \mathbf{y}}\right)=0$.

where $\mathbf{y}$ is the observation point. The matrix $(K)$ characterizing $\mathbf{k}$ in a given reference frame has coefficients $k_{i j},(i=1 . .3, j=1 . .3)$ complying with symmetry relations [9]: $k_{i j}=k_{j i}$. The coefficients $r_{i j}$ of the resistivity tensor $\mathbf{r}=\mathbf{k}^{-1}$ have similar properties.

As the tensor $\mathbf{k}$ is symmetric, it has a diagonal form when the coordinate axes are chosen as being parallel to its eigenvectors. Its eigenvalues $k_{i}$ and determinant $|\mathbf{k}|$ are strictly positive. As a consequence, it is possible to define the square root $\mathbf{k}^{1 / 2}$ of $\mathbf{k}$ that is also symmetric and definite positive [15], having eigenvalues that are the square roots of the eigenvalues of $\mathbf{k}$ and eigenvectors identical to those of $\mathbf{k}$. This square root has an inverse $\mathbf{k}^{-1 / 2}$ that is also symmetric positive definite. The conductivity tensor is denoted by $\mathbf{k}$ for $3 \mathrm{D}$ cases ( $\mathbf{k}_{2}$ for 2D case). The 2D cases may correspond to two kinds of plane problems related to different physical situations: "plane gradient" or "plane flux". In the following, only the case of "plane gradient" will be used. It corresponds to a long domain in the direction orthogonal to the plane. In this case, the gradient of $u$ is parallel to the plane $\mathbf{P}=\left(O, y_{1}, y_{2}\right)$ [12]. The 2D conductivity tensor is represented by a $2 \times 2$ matrix with $k_{i j}, i=1 . .2, j=1 . .2$ whose coefficients are the same as the ones of 3D conduction in the chosen plane.

\section{2. $2 D$ and 3D Green's functions for anisotropic conduction}

We recall now the classical expression of Green's functions for general anisotropic media [2,14,16]; this Green's function, $G(\mathbf{x}, \mathbf{y})$, gives the influence of a unit source of heat at the source point $(\mathbf{x})$ on the temperature at the observation point (y). We choose here the compact and general formulation using conductivity tensor [2] for the 3D case:

$G(\mathbf{x}, \mathbf{y})=\frac{1}{4 \pi|\mathbf{k}|^{1 / 2}} \frac{1}{\bar{r}}$

where:

$\bar{r}^{2}=(\mathbf{y}-\mathbf{x}) \cdot \mathbf{k}^{-1} \cdot(\mathbf{y}-\mathbf{x})$.

and $|\mathbf{k}|$ is the determinant of the conductivity tensor. A constant $C$ could be added to (3), but if we consider the usual condition $u=0$ at infinity, then we have $C=0$. For plane problems, the Green's function is (e.g. [2]):

$G_{2}(\mathbf{x}, \mathbf{y})=\frac{1}{2 \pi\left|\mathbf{k}_{2}\right|^{1 / 2}} \ln \left(\frac{\lambda}{\left|\mathbf{k}_{2}\right|^{1 / 4} \bar{\Gamma}_{2}}\right)$.

where $\lambda$ is the characteristic length, and appears as an unknown constant, and $\bar{r}_{2}$ is obtained by replacing $\mathbf{k}$ by $\mathbf{k}_{2}$ into Eq. (4). The term $\left|\mathbf{k}_{2}\right|^{1 / 4}$ has been introduced to ensure that the physical dimension of $\lambda$ is a length. It is not possible to choose $\lambda$ such that the Green's function tends to zero at infinity.

The characteristic length $\lambda$ is obtained, when looking for the 2D Green's function by integration of the 3D Green's function, as for the isotropic case. The discussion of the choice of this length is one of the main objectives of this paper. Indeed, the choice of this length can have two consequences:

- Degenerate scale 
When writing the BIE by using the 2D Green's function, a degenerate scale can appear. This degenerate scale depends on $\lambda$. This is a wellknown problem for Laplace equation [1], but it will be seen thereafter in Section 4 that anisotropic conduction can lead also to a degenerate scale.

\section{- Influence on the solution}

For interior problems, it can be shown (as for the isotropic case in [3]) that the choice of $\lambda$ does not affect the result, as soon as the scale is not degenerate. However, for exterior problems, if the total flux is not null, the solution depends on $\lambda$. A simple example for an isotropic material is the case of the exterior problem related to a cylinder with a circular cross section $C$ of radius $R$ : if the potential given at the boundary is constant, equal to $u_{0}$, the normal flux is also constant at the boundary and the resultant of the normal flux along the boundary is given by:

$\int_{C} \mathbf{q n} d s=\frac{2 \pi \cdot u_{0}}{\ln \left(\frac{\lambda}{R}\right)}$

which is not null and depends on $\lambda$. It can be troublesome to see that the integral of the normal flux over the boundary surface is not null, which seems contrary to the conservation of energy. As shown in [3], the $2 D$ solution provides an approximation of the $3 D$ solution of the exterior problem related to a finite cylinder with a constant normal flux at its lateral boundary. This exterior problem is the limit when $R_{1}$ tends to infinity of an interior problem whose boundary $C_{1}$ is the surface of the finite cylinder, from one hand, completed by the surface of a sphere $S_{1}$ whose radius $R_{1}$ is large compared to the dimensions of the cylinder, from another hand. From the conservation of energy, the integral of the exterior normal flux over $C_{1}$ is null. It means that the integral of the flux over the surface of the cylinder is exactly equilibrated by the integral of the flux over the external sphere. Obviously, the conservation of energy is also met at the limit of an infinite value of $R_{1}$.

The problem to find the convenient value of $\lambda$ in the case of isotropic materials has been studied in [3]. The choice of $\lambda$ has been discussed in the case of "plane gradient" defined before, i.e. for domains being bounded internally by cylinders that are very large along one dimension: in this case, the results depend on $\lambda$. It has been shown in the isotropic case that the optimal choice of $\lambda$ for domains that are long along direction $\mathbf{e}_{3}$ is simply $\lambda=2 a$, where $2 a$ is the length of the internal cylinder [3]. This optimal choice provides a correct approximation of the 3D solution in the median plane of the cylinder in the outer vicinity of the cylindrical boundary. In this case, the optimal Green's function is provided by a segment source of length $2 a$.

In the following section, we will focus on the determination of the field generated by a segment source. In section 5.3 , the question to know if this segment source corresponds to an "optimal" value of $\lambda$ will be answered.

\section{Segment sources in anisotropic media}

We choose an orthonormal basis $\left(\mathbf{e}_{1}, \mathbf{e}_{2}, \mathbf{e}_{3}\right)$ such that $\mathbf{e}_{3}$ has the direction of the segment source (Fig. 2). The plane defined by $y_{3}=0$ is denoted by P.

\subsection{Computation of the field $\widetilde{G}_{a}$ generated by the segment source}

The segment source is the set $\mathbf{x}+\epsilon \mathbf{e}_{3}$ with $€ \in[-a, a]$. Next, $\widetilde{G}_{a}$, the field generated by the segment source of total length $2 \mathrm{a}$ at $\mathbf{y}$ is defined as:

$\widetilde{G}_{a}(\mathbf{x}, \mathbf{y})=$

$\frac{1}{4 \pi|\mathbf{k}|^{1 / 2}} \int_{-a}^{a} \frac{\mathrm{d} \epsilon}{\sqrt{\left(\mathbf{x}+\epsilon \mathbf{e}_{3}-\mathbf{y}\right) \cdot \mathbf{k}^{-1} \cdot\left(\mathbf{x}+\epsilon \mathbf{e}_{3}-\mathbf{y}\right)}}$
The term under square root can be expressed as:

$$
\begin{aligned}
\left(x+e_{3}-\mathbf{y}\right) \cdot \mathbf{k}^{-1} \cdot\left(\mathbf{x}+\epsilon \mathbf{e}_{3}-\mathbf{y}\right)= & \left(\epsilon \sqrt{\mathbf{e}_{3} \cdot \mathbf{k}^{-1} \cdot \mathbf{e}_{3}}+\frac{(\mathbf{x}-\mathbf{y}) \cdot \mathbf{k}^{-1} \cdot \mathbf{e}_{3}}{\sqrt{\mathbf{e}_{3} \cdot \mathbf{k}^{-1} \cdot \mathbf{e}_{3}}}\right)^{2} \\
& \left.+(\mathbf{x}-\mathbf{y}) \cdot \mathbf{K}^{-1} \cdot(\mathbf{x}-\mathbf{y})-\frac{\left((\mathbf{x}-\mathbf{y}) \cdot \mathbf{k}^{-1} \cdot \mathbf{e}_{3}\right)^{2}}{\mathbf{e}_{3} \cdot \mathbf{k}^{-1} \cdot \mathbf{e}_{3}}\right) \\
& =(\epsilon b+c)^{2}+d^{2}
\end{aligned}
$$

where:

$b=\sqrt{\mathbf{e}_{3} \cdot \mathbf{k}^{-1} \cdot \mathbf{e}_{3}}$,

$c=\left(\frac{(\mathbf{x}-\mathbf{y}) \cdot \mathbf{k}^{-1} \cdot \mathbf{e}_{3}}{\sqrt{\mathbf{e}_{3} \cdot \mathbf{k}^{-1} \cdot \mathbf{e}_{3}}}\right)$,

$d=\sqrt{\left((\mathbf{x}-\mathbf{y}) \cdot \mathbf{k}^{-1} \cdot(\mathbf{x}-\mathbf{y})-\frac{\left((\mathbf{x}-\mathbf{y}) \cdot \mathbf{k}^{-1} \cdot \mathbf{e}_{3}\right)^{2}}{\mathbf{e}_{3} \cdot \mathbf{k}^{-1} \cdot \mathbf{e}_{3}}\right)}$.

We see easily that the quantity under square root in Eq. (11) is positive or null, because the quantity defined in Eq. (8) is always positive or null. If one substitutes $\epsilon=-(\mathbf{x}-\mathbf{y}) \cdot \mathbf{k}^{-1} \cdot \mathbf{e}_{3} /\left(\mathbf{e}_{3} \cdot \mathbf{K}^{-1} \cdot \mathbf{e}_{3}\right)$ in Eq. (8), we get that:

$\left((\mathbf{x}-\mathbf{y}) \cdot \mathbf{k}^{-1} \cdot(\mathbf{x}-\mathbf{y})-\frac{\left((\mathbf{x}-\mathbf{y}) \cdot \mathbf{K}^{-1} \cdot \mathbf{e}_{3}\right)^{2}}{\mathbf{e}_{3} \mathbf{k}^{-1} \cdot \mathbf{e}_{3}}\right) \geq 0$ due to the positivity of $\mathbf{k}^{-1}$. If $0 \neq \mathbf{x}-\mathbf{y} \in \mathbf{P}$, the vector $\left(\mathbf{x}+\epsilon \mathbf{e}_{3}-\mathbf{y}\right)$ is non-null and the inequality is strict because $\mathbf{K}^{-1}$ is positive definite.

We now need to evaluate the integral:

$I=\int_{-a}^{a} \frac{\mathrm{d} \epsilon}{\sqrt{(\epsilon b+c)^{2}+d^{2}}}$.

We use the new variable $\eta=\frac{c b+c}{d}$ and we find:

$I=\frac{1}{b} \int_{\frac{-a b+c}{d}}^{\frac{a b+c}{d}} \frac{\mathrm{d} \eta}{\sqrt{1+\eta^{2}}}=\frac{1}{b}\left[\left.\ln \left(\eta+\sqrt{1+\eta^{2}}\right)\right|_{\frac{-a b+c}{d}} ^{\frac{a b+c}{d}}\right.$

3.2. Asymptotic behavior for large values of a

For $\quad \eta \gg 1, \quad \ln \left(\eta+\sqrt{1+\eta^{2}}\right) \simeq \ln (2 \eta) \quad$ and for $\quad \eta \ll-1$, $\ln \left(\eta+\sqrt{1+\eta^{2}}\right)=\ln \left(\eta-\eta \sqrt{1+1 / \eta^{2}}\right) \simeq \ln \left(\eta-\eta\left(1+1 / 2 \eta^{2}\right)\right) \quad$ and $\simeq-\ln (-2 \eta)$
we finally conclude that:

$I \simeq \frac{1}{b}\left(\ln \left(2 \frac{a b-c}{d}\right)+\ln \left(2 \frac{a b+c}{d}\right)\right)$.

For $a \gg \frac{|c|}{b}$ and $a \gg \frac{d}{b}$, we have:

$I \simeq \frac{2}{b}\left(\ln \left(2 \frac{a b}{d}\right)\right)$

leading to the asymptotic expression $G_{a}$ of $\widetilde{G}_{a}$ for large values of $a$ :

$G_{a}=\frac{1}{2 \pi b|\mathbf{k}|^{1 / 2}}\left(\ln \left(\frac{2 a b}{d}\right)\right)$

\subsection{Computation of the constant $\mathrm{d}$}

In order to express the value of $d$, let us introduce the linear application, $\mathbf{f}$, that is defined as follows:

$\mathbf{f}: \mathbf{v} \rightarrow \mathbf{k}^{-1}(\mathbf{v})-\frac{\mathbf{v} \cdot \mathbf{k}^{-1} \cdot \mathbf{e}_{3}}{\mathbf{e}_{3} \cdot \mathbf{k}^{-1} \cdot \mathbf{e}_{3}} \mathbf{k}^{-1}\left(\mathbf{e}_{3}\right)$

Evaluating the product $\mathbf{v} \cdot \mathbf{f}(\mathbf{v})$ gives: 
$\mathbf{v} \cdot \mathbf{f}(\mathbf{v})=\mathbf{v} \cdot\left(\mathbf{k}^{-1}(\mathbf{v})-\frac{\mathbf{v} \cdot \mathbf{k}^{-1} \cdot \mathbf{e}_{3}}{\mathbf{e}_{3} \cdot \mathbf{k}^{-1} \cdot \mathbf{e}_{3}} \mathbf{k}^{-1}\left(\mathbf{e}_{3}\right)\right)=\mathbf{v} \cdot \mathbf{k}^{-1}(\mathbf{v})-\frac{\left(\mathbf{v} \cdot \mathbf{k}^{-1} \cdot \mathbf{e}_{3}\right)^{2}}{\mathbf{e}_{3} \cdot \mathbf{k}^{-1} \cdot \mathbf{e}_{3}}$

and we conclude from Eq. (11):

$d=\sqrt{(\mathbf{x}-\mathbf{y}) \cdot \mathbf{f}(\mathbf{x}-\mathbf{y})}$.

The application $\mathbf{f}$ can be restricted to an application $\mathbf{f}_{p}$ from $\mathbf{P} \rightarrow \mathbf{P}$; indeed, if $\mathbf{v} \in \mathbf{P}$, then

$\mathbf{e}_{3} \mathbf{f}(\mathbf{v})=\mathbf{e}_{3} \cdot \mathbf{k}^{-1}(\mathbf{v})-\frac{\mathbf{v} \cdot \mathbf{k}^{-1} \cdot \mathbf{e}_{3}}{\mathbf{e}_{3} \cdot \mathbf{k}^{-1} \cdot \mathbf{e}_{3}} \mathbf{e}_{3} \cdot \mathbf{k}^{-1} \cdot \mathbf{e}_{3}=0$

As the plane $\mathbf{P}$ is orthogonal to $\mathbf{e}_{3}$, we conclude that $\mathbf{f}(\mathbf{v}) \in \mathbf{P}$. Now, it will be shown that $\mathbf{f}_{p}$ can be expressed simply from the conductivity tensor. We denote by $\mathbf{p}$ the orthogonal projection on $\mathbf{P}$. We prove now that $\mathbf{f}_{P}$ is the inverse of the restriction of $\mathbf{p} \times \mathbf{k}$ to $\mathbf{P}$.

$(\mathbf{p} \circ \mathbf{k}) \mathbf{f}_{P}(\mathbf{v})=\mathbf{p} \circ \mathbf{k}\left(\mathbf{k}^{-1}(\mathbf{v})-\frac{\mathbf{u} \cdot \mathbf{k}^{-1} \cdot \mathbf{e}_{3}}{\mathbf{e}_{3} \cdot \mathbf{k}^{-1} \cdot \mathbf{e}_{3}} \mathbf{k}^{-1}\left(\mathbf{e}_{3}\right)\right)=\mathbf{p}(\mathbf{v})-\frac{\mathbf{v} \cdot \mathbf{k}^{-1} \cdot \mathbf{e}_{3}}{\mathbf{e}_{3} \cdot \mathbf{k}^{-1} \cdot \mathbf{e}_{3}} \mathbf{p}\left(\mathbf{e}_{3}\right)$.

For $\mathbf{v} \in \mathbf{P}$, we have $\mathbf{p}(\mathbf{v})=\mathbf{v}$ and $\mathbf{p}\left(\mathbf{e}_{3}\right)=0$, and we can conclude that $\forall \mathbf{v} \in \mathbf{P},(\mathbf{p} \circ \mathbf{k}) \circ \mathbf{f}_{p}(\mathbf{v})=\mathbf{v}$.

This proves finally that $\mathbf{f}_{p}$ is the inverse of the restriction $\mathbf{k}_{2}$ of $\mathbf{p} \cdot \mathbf{k}$ to $\mathbf{P}$. The matrix $\left(K_{2}\right)$ of $\mathbf{k}_{2}$ contains only the components related to $y_{1}, y_{2}$. Finally the expression of $d$ is given by:

$d=\sqrt{(\mathbf{x}-\mathbf{y}) \cdot \mathbf{k}_{2}^{-1} \cdot(\mathbf{x}-\mathbf{y})}$.

\subsection{Computation of the constant $\mathrm{b}$}

The constant $b$ is given by $b=\sqrt{\mathbf{e}_{3} \cdot \mathbf{k}^{-1} \cdot \mathbf{e}_{3}}=\sqrt{r_{33}}$, where $r_{33}$ comes from the matrix characterizing the resistivity tensor. From classical results of linear algebra, this term is given using the cofactor $\mathrm{C}_{33}$ of the term $k_{33}$ in the conductivity matrix by:

$r_{33}=\frac{\mathrm{C}_{33}}{|\mathbf{k}|}$.

The cofactor being the determinant of $\mathbf{k}_{2}$, it leads to

$b=\sqrt{\frac{\left|\mathbf{k}_{2}\right|}{|\mathbf{k}|}}$.

Finally, substituting $d$ and $b$ into the expression (16) of $G_{a}$ leads to:

$G_{a}(\mathbf{x}, \mathbf{y})=\frac{1}{2 \pi\left|\mathbf{k}_{2}\right|^{1 / 2}} \ln \left(\frac{2 a \sqrt{\mathbf{e}_{3} \cdot \mathbf{k}^{-1} \cdot \mathbf{e}_{3}}}{\sqrt{(\mathbf{x}-\mathbf{y}) \cdot \mathbf{k}_{2}^{-1} \cdot(\mathbf{x}-\mathbf{y})}}\right)$.

This is exactly the 2D Green's function defined by Eq. (52) where the length $\lambda$ is given by:

$\lambda=2 a \sqrt{\mathbf{e}_{3} \cdot \mathbf{K}^{-1} \cdot \mathbf{e}_{3}}\left|\mathbf{k}_{2}\right|^{1 / 4}$

4. Use of a linear transformation to change the anisotropic problem into an isotropic one

As explained previously, the solution of a plane conduction problem by the Boundary Element Method in the isotropic case provides an approximation of the solution of a 3D problem outside a long cylinder if the characteristic length in the 2D Green's function is equal to the length of the cylinder [3]. To study the anisotropic case, it is useful to consider that the solution of any homogeneous anisotropic conductivity problem can be provided by solving an associated problem related to isotropic conductivity. Several papers were based on this important property [5,16-23]. In a first step, the features of the transformation that is used to build an associated isotropic problem from an anisotropic one are given. The relations between boundary conditions are next studied and the relations between boundary integral equations and Green's functions for both problems are provided. It will allow us to study the degenerate scales for the plane anisotropic conduction problems and finally to provide the plane approximation of $3 \mathrm{D}$ problems related to long cylinders.

\subsection{Domain transformation and the solution of an anisotropic} conduction $B V P$

4.1.1. Domain transformation for $3 D$ and $2 D$ BVPs

As the conductivity tensor $\mathbf{k}$ is symmetric and positive definite, it has a (principal) square root $\mathbf{k}^{1 / 2}$ with its inverse $\mathbf{k}^{-1 / 2}$, both being symmetric and positive definite (see Section 2.1). For 3D problems, we define a linear transformation $\mathbf{T}$ :

$\mathbf{T}(\mathbf{y})=\mathbf{y}^{\prime}=\sqrt{k} \mathbf{K}^{1 / 2} \mathbf{y}$

where $k=|\mathbf{k}|^{1 / 3}$. The transformation is given here in a concise form that can be found when using a rectangular coordinate system corresponding to principal directions in [12]. The introduction of the term $\sqrt{k}$ that was not generally used in earlier formulations of this transformation, allows us to ensure the consistence of physical units between original space and transformed space. A consequence of this choice is that the determinant of $\mathbf{T}$ is $|\mathbf{T}|=1$. For $2 \mathrm{D}$ problems, the physical homogeneity is restored by a similar transformation, replacing $k$ by $\sqrt{\left|\mathbf{k}_{2}\right|}$ and $\mathbf{k}$ by $\mathbf{k}_{2}$

If $u$ is a solution of Eq. (2) on a domain $D$, one can define the following function on $D^{\prime}=\mathbf{T}(D)$ :

$u^{\prime}\left(\mathbf{y}^{\prime}\right)=u(\mathbf{y})$,

where $\mathbf{y}^{\prime}=\mathbf{T}(\mathbf{y})$. Combining Eqs. (2) and (29) while using a basis where $\mathbf{k}$ is diagonal, it comes readily that: $\Delta_{y^{\prime}} u^{\prime}=0$. It means that the function $u^{\prime}$ is a harmonic function of $\mathbf{y}^{\prime}$, and can be associated with an isotropic conduction problem over $D^{\prime}$ with conductivity

$k=|\mathbf{k}|^{1 / 3}$

(or $k_{2}=\sqrt{\left|\mathbf{k}_{2}\right|}$ for 2D problem).

4.1.2. Relations between isotropic and anisotropic $B V P$

One considers now the BVP on $u$ within $D$ with Dirichlet condition over $\partial D_{u}$ and a heat flux condition over $\partial D_{q}$, that can be written:

$\left\{u(\mathbf{y})=u_{0} \quad \mathbf{y} \in \partial D_{u}\right.$

$q(\mathbf{y})=\mathbf{q} \cdot \mathbf{n}=q_{0} \quad \mathbf{y} \in \partial D_{q}$

where $u_{0}$ and $q_{0}$ are known boundary functions and $\mathbf{n}$ is the outer unit normal at $\mathbf{y}$. This problem can be turned into a BVP on $u^{\prime}$ defined over $D^{\prime}$.

If Dirichlet's boundary conditions are concerned, it comes readily that $u^{\prime}=u_{0}$ on $\partial D_{u^{\prime}}$, where $\partial D_{u^{\prime}}{ }^{\prime}$ is the transform of $\partial D_{u}$ by $\mathbf{T}$.

For Neumann boundary conditions, the relation is not so easy to establish. It will necessitate to write the transformation of oriented surface elements, fluxes and normal fluxes by $\mathbf{T}$. We give here a tensor formulation [13, 23] ; a coordinate formulation can be found in [22].

Transformation of oriented surface elements

Let us consider the boundary $\partial D$ of the original domain. The transformation of surface elements can be performed by using a mapping of the surface $\partial D$ through a parametric representation. The parametric representation of the surface uses a function $g$ that associates to curvilinear coordinates $\xi, \eta$ (chosen in a reference plane) the point $\mathbf{y}$ :

$\mathbf{y}=\mathbf{g}(\xi, \eta)$.

Let us consider the oriented surface element $\mathrm{d} \mathbf{S}$ around point $\mathbf{y}$ given by $\mathrm{d} \mathbf{S}=\mathbf{n d} \boldsymbol{S}$ where $\mathbf{n}$ is the outer unit normal to the surface at $\mathbf{y}$ and $d S$ is the surface element.

With a convenient choice of the order of $\xi, \eta$ (to recover the same 
orientation for $\mathbf{n}$ and $\mathbf{d S}$ ), $\mathbf{d S}$ can be obtained from $\mathbf{g}$ by:

$\mathrm{d} \mathbf{S}=\frac{\partial \mathrm{g}}{\partial \xi} \times \frac{\partial \mathbf{g}}{\partial \eta} d \xi d \eta=\mathbf{J} d \xi d \eta$,

where the cross product $\mathbf{J}$ is computed from the components of the Jacobian matrix(e.g. [24]) of the transformation $\xi, \eta \rightarrow y_{1}, y_{2}, y_{3}$.

Then, $\mathbf{T}$ transforms $\mathbf{y}$ into $\mathbf{y}^{\prime}$ (Eq. (28)) and therefore we define:

$\mathbf{g}^{\prime}(\xi, \eta)=\mathbf{T} \cdot \mathbf{g}(\xi, \eta)$,

with $\mathbf{g}^{\prime} \in \partial D$. Using the symmetry of $\mathbf{T}$ and the property $|\mathbf{T}|=1$, the computation of the vector product leads to

$\frac{\partial \mathbf{g}}{\partial \xi} \times \frac{\partial \mathbf{g}}{\partial \eta}=\mathbf{T} \cdot\left(\frac{\partial \mathbf{g}^{\prime}}{\partial \xi} \times \frac{\partial \mathbf{g}^{\prime}}{\partial \eta}\right)$,

And finally, we get the following special case of Nanson's formula:

$\mathrm{d} \mathbf{S}=\mathbf{T} \cdot \mathrm{d} \mathbf{S}^{\prime}$

We denote by $\mathbf{J}^{\prime}$ the cross product $\frac{\partial \mathbf{g}^{\prime}}{\partial \xi^{\xi}} \times \frac{\partial \mathbf{g}^{\prime}}{\partial \eta}$. From the last result, the ratio between the scalar surface elements is obtained using the moduli of the cross products $J=\left|J^{\prime}\right|$ and $J^{\prime}=\left|J^{\prime}\right|$ by: $d S^{\prime} / d S=J^{\prime} / J$.

Transformation of the fluxes and of the elementary product $q . d S$

Using $\mathbf{k} \cdot \mathbf{T}=\mathbf{T} \cdot \mathbf{k}$ and $\mathbf{q}^{\prime}=k \partial u^{\prime} / \partial \mathbf{y}^{\prime}$, we get the following relation:

$\mathbf{q}=\mathbf{k} \cdot \frac{\partial u}{\partial \mathbf{y}}=\mathbf{k} \cdot \frac{\partial \mathbf{y}^{\prime}}{\partial \mathbf{y}} \cdot \frac{\partial u^{\prime}}{\partial \mathbf{y}^{\prime}}=\mathbf{k} \cdot \mathbf{T} \cdot \frac{\partial u^{\prime}}{\partial \mathbf{y}^{\prime}}=\frac{\mathbf{k} \cdot \mathbf{T}}{k} \cdot \mathbf{q}^{\prime} \cdot$

Using that $\mathbf{k} \cdot \mathbf{T} / k=\mathbf{k} \cdot \sqrt{k} \mathbf{k}^{-1 / 2} / k=\mathbf{k}^{1 / 2} / \sqrt{k}=\mathbf{T}^{-1}$ in (37) we finally get:

$\mathbf{q}=\mathbf{T}^{-1} \cdot \mathbf{q}^{\prime}$

Combining this relation with the transformation of $\mathbf{d S}$ leads to

$q \mathrm{~d} S=\mathbf{q} \cdot \mathrm{d} \mathbf{S}=\mathbf{q}^{\prime} \cdot \mathrm{d} \mathbf{S}^{\prime}=q^{\prime} \mathrm{d} S^{\prime}$.

It should be noted that the proof of the preceding equality relies on the fact that $\mathbf{T}$ and $\mathbf{k}$ are symmetric, positive definite. Finally, the Neumann boundary conditions in the transformed domain are obtained by combining this result with the transformation of surface elements, leading to: $q^{\prime}=q J / J^{\prime}$. To conclude, $u^{\prime}$ is the solution of the BVP related to Laplace equation within domain $D^{\prime}$ with boundary conditions $u^{\prime}=u_{0}$ on $\partial D_{u}^{\prime}$, transform of $\partial D_{u}$, and $q^{\prime}=q_{0} J / J^{\prime}$ on $\partial D_{q}^{\prime}$, transform of $\partial D_{q}$.

4.2. Related BIEs for exterior problems in the original and transformed domains

The previous results allow us to produce the relation between the BIEs in the original and transformed domains and, in particular, to recover the Green's function for anisotropic problems. Let us consider the 3D BIE in the initial domain (with anisotropy), in the regularized form and for exterior problems, as described in [2]:

$u(\mathbf{x})+\int_{a D}(u(\mathbf{y})-u(\mathbf{x})) q(G(\mathbf{x}, \mathbf{y})) \mathrm{d} S-\int_{\partial D} q(u(\mathbf{y})) G(\mathbf{x}, \mathbf{y}) \mathrm{d} S=0$,

with $q(G)=\left[\left(\mathbf{k} \cdot \frac{\partial G}{\partial y}\right) \cdot \mathbf{n}\right]$ and a similar relation for $q(u)$.

Using the fact that $q(G)$ is transformed as $q(u)$ and Eq. (39), it comes:

$u^{\prime}\left(\mathbf{x}^{\prime}\right)+\int_{\partial D^{\prime}}\left(u^{\prime}\left(\mathbf{y}^{\prime}\right)-u^{\prime}\left(\mathbf{x}^{\prime}\right)\right) q^{\prime}\left(G^{\prime}\left(\mathbf{x}^{\prime}, \mathbf{y}^{\prime}\right)\right) \mathrm{d} S^{\prime}-\int_{\partial D^{\prime}} q^{\prime}\left(u^{\prime}\left(\mathbf{y}^{\prime}\right)\right) G^{\prime}\left(\mathbf{x}^{\prime}, \mathbf{y}^{\prime}\right)$

$\mathrm{d} S^{\prime}=0$,

where

$G^{\prime}\left(\mathbf{x}^{\prime}, \mathbf{y}^{\prime}\right)=G(\mathbf{T} \cdot \mathbf{x}, \mathbf{T} \cdot \mathbf{y})$.

and $q^{\prime}\left(G^{\prime}\right)=k \frac{\partial G^{\prime}}{\partial \sigma^{\prime}} \cdot \mathbf{n}$ with a similar relation for $q^{\prime}\left(u^{\prime}\right)$. It is then easy to recover the 3D Green's function for anisotropic conduction (Eq. (3)) from Eq. (42). A similar 2D transformation produces the same result for $2 \mathrm{D}$ problems.

4.3. Application to the degenerate scale for anisotropic $2 D$ problems

Degenerate scales are well known for 2D Laplace equation. One considers the single layer operators for Laplace equation related to diverse homothetic plane contours $\rho \Gamma^{\prime}, \rho$ being a positive scalar, that writes:

$\int_{\rho \Gamma^{\prime}} \frac{1}{2 \pi} \ln \left(\left\|\mathbf{x}^{\prime}-\mathbf{y}^{\prime}\right\|\right) \phi\left(\mathbf{y}^{\prime}\right) \mathrm{d} S^{\prime}=f\left(\mathbf{x}^{\prime}\right)$,

where $\Gamma^{\prime}$ and $f$ comply with suitable regularity conditions.

For given $f$, these integral equations have only one solution, except for a specific value $\rho=\rho_{0}$. For this value, the related integral equation degenerates and has an infinite number of solutions. The non-dimensional constant $\rho_{0}$ is customarily called the "degenerate scale". When the boundary is at a degenerate scale, it is easily seen that the integral equation for $f=0$ has multiple solutions.

For a given boundary, the degenerate scale is related to the logarithmic capacity $C_{\Gamma}$ of the contour by $\rho_{0}=1 / C_{T}$. The logarithmic capacity is well documented, that provides the degenerate scale for numerous contours [25]. It has also been shown in [3] that, in addition to the necessity of avoiding singular integral equations, energetic considerations induce that $\rho$ must comply with the inequality $\rho<\rho_{0}$.

From the previous results, performing the transformation $\mathbf{T}^{-1}$ on the single layer operator leads to the single layer operator for the anisotropic conduction with $\lambda=1$.

This relation proves that if $\rho \Gamma^{\prime}$ is at the critical scale, the single layer integral equation for the anisotropic problem involving the Green's function $G_{2}(\mathbf{x}, \mathbf{y})$ (with $\left.\lambda=1\right)$ has also multiple solutions and is therefore at the critical scale.

So, the critical scale for $\Gamma$ and the Green's function $G_{2}$ with $\lambda=1$ is the same as the critical scale for the transformed contour $\Gamma^{\prime}$ and the Green's function for the Laplace operator. It allows us the determination of the degenerate scale in the anisotropic case. An example of determination of degenerate scale will be shown in section 6 .

\section{Plane approximation of a 3D anisotropic problem}

Let us now consider a very long cylindrical domain $D$ having length $2 a$ along $y_{3}$ and whose bottom and top surfaces are located in the planes $y_{3}= \pm a$, perpendicular to the axis of the cylinder, (i.e. a right cylinder). The boundary of its intersection with the plane $O y_{1} y_{2}$ is the curve $\Gamma$ (Fig. 3).

The general case corresponds to a domain $D$ outside a long cylinder whose axis is oriented along an arbitrary direction of the space with respect to the eigenvectors of $\mathbf{k}$. Its transform $D^{\prime}$ by $\mathbf{T}$ is also located outside a long cylinder. However, it is generally no more a right cylinder: it is an oblique cylinder, its sections at top and bottom being not perpendicular to the long direction. The domain outside the long cylinder in the transformed domain can also be studied by using a plane approximate solution in an orthogonal section around its median plane.

The problem outside a long cylinder can be approximated in its

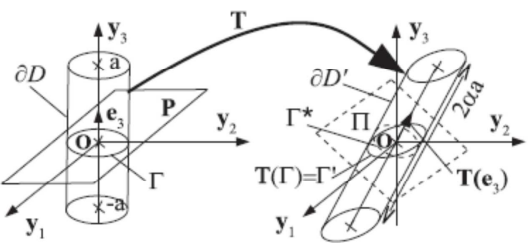

Fig. 3. Geometric notations for a long cylindrical domain in an anisotropic domain (left) and its transform (right). 
median plane by a 2D solution of "plane gradient" type. In the first step, it will be shown that if the problem in the original domain is of "plane gradient" type in its normal section outside contour $\Gamma$, the problem in the transformed domain is also of "plane gradient" in a normal section outside the cylinder in the transformed domain.

Next, the relation between plane integral equations in original and transformed domains will be established. Finally, these results will be used to obtain the value of the constant $\lambda$ to be introduced into the Green's function.

\subsection{Transformation of a potential of "plane gradient" type}

One considers a potential of "plane gradient" type in the original domain corresponding to plane $\mathbf{P}$ (orthogonal to the direction $\mathbf{e}_{3}$ parallel to the long direction of the cylinder). It is therefore characterized by:

$\frac{\partial u}{\partial \mathbf{y}} \cdot \mathbf{e}_{3}=0$,

Using $\frac{\partial u}{\partial y}=\sqrt{k} \mathbf{K}^{-1 / 2} \frac{\partial u}{\partial y^{\prime}}$ and the symmetry of $\mathbf{K}^{-1 / 2}$, this can be written:

$\left[\sqrt{k} \mathbf{k}^{-1 / 2} \cdot \frac{\partial u}{\partial \mathbf{y}^{\prime}}\right] \cdot \mathbf{e}_{3}=\frac{\partial u}{\partial \mathbf{y}^{\prime}} \cdot\left[\sqrt{k} \mathbf{k}^{-1 / 2} \cdot \mathbf{e}_{3}\right]=\frac{\partial u}{\partial \mathbf{y}^{\prime}} \cdot \mathbf{v}^{\prime}=0$

where $\mathbf{v}^{\prime}=\sqrt{k} \mathbf{k}^{-1 / 2} \cdot \mathbf{e}_{3}$ is the transform of $\mathbf{e}_{3}$ by $\mathbf{T}$ and is parallel to the axis of the cylinder in the transformed domain. Finally, the potential in the transformed domain is also of "plane gradient" type in the plane $\Pi$ perpendicular to $\mathbf{v}^{\prime}$

5.2. Relation between BIEs in the original and transformed domains

5.2.1. Transformation between normal sections of the cylinders in original and transformed domains; choice of the boundary $\Gamma^{*}$ of the $2 D$ isotropic approximate problem

One considers now the plane BIE for the exterior problem related to the original plane domain that is located outside the normal section of the bounding cylinder. This problem is of "plane gradient" type within $\mathbf{P}$ and the related integral equation writes:

$u(\mathbf{x})+\int_{\Gamma}(u(\mathbf{y})-u(\mathbf{x})) q\left(G_{2}(\mathbf{x}, \mathbf{y})\right) d s-\int_{\Gamma} q(u(\mathbf{y})) G_{2}(\mathbf{x}, \mathbf{y}) d s=0$

where $G_{2}$ is the Green's function for the plane anisotropic domain given by Eq. (52).

When performing the transform $\mathbf{T}$, all points $\mathbf{y}$ of $\mathbf{P}$ are tranformed into points $\mathbf{y}^{\prime}$ of $\mathbf{P}^{\prime}=\mathbf{T}(\mathbf{P})$. In the general case, $\mathbf{P}^{\prime}$ is not orthogonal to the axis of the cylinder in the transformed domain. However, it can be noticed that in the vicinity of the median plane of the cylinder in the transformed domain, the field is of "plane gradient" type and as a consequence, the potential does not depend on the position along a line parallel to the axis of the cylinder. It means that the potential complies with:

$u\left(\mathbf{y}^{\prime}\right)=u\left(\pi\left(\mathbf{y}^{\prime}\right)\right)=u\left(\mathbf{y}^{*}\right)$.

where $\mathbf{y}^{*}=\pi\left(\mathbf{y}^{\prime}\right)$ and $\pi$ is the projection onto the median plane $I$ orthogonal to the axis of the cylinder in the transformed domain. There is a one to one correspondence between points $\mathbf{y}$ of $\mathbf{P}$ and $\mathbf{y}^{*}$ of $\Pi$ through points of $\mathbf{P}^{\prime}$.

As a consequence, the plane BIE within the normal plane $\Pi$ in the transformed domain can be obtained by transforming the BIE within $\mathbf{P}$ by $\mathbf{T}^{*}$ the restriction of $\mathbf{T} \circ \pi$ to $\mathrm{P}$. The one to one correspondence between $\mathbf{y}$ and $\mathbf{y}^{*}$ implies that $\mathbf{T}^{*}$ is invertible. To obtain the expression of the transformed BIE, it is now necessary to find the relations in original and transformed planes $P$ and $\Pi$ of

- the elements of integration $q d s$ and $q^{*} d s^{*}$.

- the boundary conditions on the curves $\Gamma$ and $\Gamma^{*}$ in both planes.
- the plane Green's functions.

5.2.2. Relation between elements of integration

It has been shown when studying the relation between 3D BIE that the normal fluxes at the boundary and the surface elements comply with $q \mathrm{~d} S=q^{\prime} \mathrm{d} S^{\prime}$ (Eq. (39)). A surface element at the boundary in the original domain is given by $\mathrm{d} S=\mathrm{d} s . \mathrm{d} y_{3}$, where $d s$ is the contour element around the normal section. This surface element is transformed into a surface element in the transformed domain that is given by $\mathrm{d} S^{\prime}=\mathrm{d} s^{*} . \mathrm{d} l$ where $\mathrm{d} s^{*}$ is the contour element around the normal section $\left(\Gamma^{\prime}\right)$ (in $\Pi$ ) and $\mathrm{d} l$ is given by the length of the vector $\mathbf{T}\left(\mathbf{e}_{3}\right) \mathrm{d} y_{3}$, that is oriented along the axis of the cylinder in the transformed

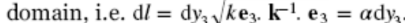

It comes that $q d s=\alpha q^{\prime} \mathrm{d} s^{*}=\alpha q^{*} \mathrm{~d} s^{*}$, with $\alpha=\sqrt{k \mathbf{e}_{3} \cdot \mathbf{K}^{-1} \cdot \mathbf{e}_{3}}$. The equality $q^{\prime}=q^{*}$, where $q^{*}$ is the normal flux for the 2D problem comes from the fact that the normal to $\Gamma^{*}$ in $\Pi$ is the same as the normal to the transformed cylinder.

The value of $\alpha$ can be computed by noticing that $\mathbf{e}_{3} \cdot \mathbf{k}^{-1} \cdot \mathbf{e}_{3}=r_{33}$ where $r_{33}$ has been found previously equal to $\frac{\mathbf{k}_{2} !}{\mathbf{k} \mid}$. Finally, $\alpha$ is given by: $\alpha=\frac{\sqrt{\left|\mathbf{k}_{2}\right|}}{k}$

\subsubsection{Relation between boundary conditions}

Dirichlet boundary conditions come directly from Eq. (47) and from the definition of Dirichlet boundary conditions on $\Gamma$. The Neumann boundary conditions are obtained by noticing that the normal flux $q$ on $\Gamma$ is the same as the normal flux on the cylinder and that the normal flux on the transformed cylinder complies with $q^{*}=q^{\prime}$ in the vicinity of the median plane. But, from the general relation in the case of the 3D problem, it comes $q J=q^{\prime} J^{\prime}=q^{*} J^{\prime}$. In the 3D case, $J$ and $J^{\prime}$ depend on two curvilinear parameters $\xi$ and $\eta$. However, in the case of the cylinder, it is natural to take one of the curvilinear coordinates as being the coordinate $\eta=y_{3}$ along the axis of the cylinder, with $y_{1}$ and $y_{2}$ being only functions of the other one, $\xi$, that is the curvilinear coordinate allowing us to describe the contour $\Gamma$. With these notations, it comes that $J$ and $J^{\prime}$ are only functions of $\xi$ given by:

$J(\xi)=|\mathbf{J}|=\left|\frac{\partial \mathbf{g}}{\partial \xi} \times \frac{\partial \mathbf{g}}{\partial \eta}\right|=\left|-\frac{\partial g_{2}}{\partial \xi} \mathbf{e}_{1}+\frac{\partial g_{1}}{\partial \xi} \mathbf{e}_{2}\right|$

$J^{\prime}(\xi)=\left|\mathbf{J}^{\prime}\right|=\left|\mathbf{T}^{-1}\left[-\frac{\partial g_{2}}{\partial \xi} \mathbf{e}_{1}+\frac{\partial g_{1}}{\partial \xi} \mathbf{e}_{2}\right]\right|$

These relations added to $q J=q^{*} J^{\prime}$ provide the Neumann's boundary condition on $\Gamma^{*}$ knowing the Neumann's boundary condition on $\Gamma$ and the mapping of $\Gamma$ :

$q^{*}=\frac{J}{J^{\prime}} q_{0}$.

\subsubsection{Relation between 2D Green's functions}

To establish the relation between the 2D Green's functions, we start from the Green's function in $\Pi$ plane. Taking into account that the conductivity in the transformed domain is $k=|\mathbf{k}|^{1 / 3}(30)$, this Green's function is given by:

$G_{2}^{*}=\frac{1}{2 \pi k} \ln \left(\frac{\lambda^{\prime}}{\sqrt{\left(\mathbf{y}^{*}-\mathbf{x}^{*}\right) \cdot\left(\mathbf{y}^{*}-\mathbf{x}^{*}\right)}}\right)=\frac{1}{2 \pi k} \ln \left(\frac{\lambda^{\prime}}{\sqrt{\pi\left(\mathbf{x}^{\prime}-\mathbf{y}^{\prime}\right) \cdot \pi\left(\mathbf{x}^{\prime}-\mathbf{y}^{\prime}\right)}}\right)$,

where $\mathbf{x}^{\prime}=\mathbf{T}(\mathbf{x}), \mathbf{y}^{\prime}=\mathbf{T}(\mathbf{y})$. At this step, the parameter $\lambda^{\prime}$ is unknown.

The projection on $\Pi$, orthogonal to $\mathbf{T}\left(\mathbf{e}_{3}\right)$ is given by:

$\pi\left(\mathbf{x}^{\prime}-\mathbf{y}^{\prime}\right)=\mathbf{x}^{\prime}-\mathbf{y}^{\prime}-\frac{\mathbf{T}\left(\mathbf{e}_{3}\right) \cdot\left(\mathbf{x}^{\prime}-\mathbf{y}^{\prime}\right)}{\left\|\mathbf{T}\left(\mathbf{e}_{3}\right)\right\|^{2}} \mathbf{T}\left(\mathbf{e}_{3}\right)$. 
Then, using Eq. (28) and the symmetry of $\mathbf{k}^{-1 / 2}$, we evaluate $\boldsymbol{\pi}\left(\mathbf{x}^{\prime}-\mathbf{y}^{\prime}\right) \cdot \boldsymbol{\pi}\left(\mathbf{x}^{\prime}-\mathbf{y}^{\prime}\right)$ :

$$
\begin{aligned}
\boldsymbol{\pi}\left(\mathbf{x}^{\prime}-\mathbf{y}^{\prime}\right) \cdot \boldsymbol{\pi}\left(\mathbf{x}^{\prime}-\mathbf{y}^{\prime}\right) & =\left(\mathbf{x}^{\prime}-\mathbf{y}^{\prime}\right) \cdot\left(\mathbf{x}^{\prime}-\mathbf{y}\right)-\frac{\left(\left(\mathbf{x}^{\prime}-\mathbf{y}^{\prime}\right) \cdot \mathbf{T}\left(\mathbf{e}_{3}\right)\right)^{2}}{\left\|\mathbf{T}\left(\mathbf{e}_{3}\right)\right\|^{2}} \\
& =k\left(\left(\mathbf{x}^{\prime}-\mathbf{y}^{\prime}\right) \cdot \mathbf{k}^{-1} \cdot\left(\mathbf{x}^{\prime}-\mathbf{y}^{\prime}\right)-\frac{\left(\left(\mathbf{x}^{\prime}-\mathbf{y}^{\prime}\right) \cdot \mathbf{k}^{-1} \cdot \mathbf{e}_{3}\right)^{2}}{\mathbf{e}_{3} \cdot \mathbf{k}^{-1} \cdot \mathbf{e}_{3}}\right) .
\end{aligned}
$$

From Eqs. (11), (23), it comes:

$(\mathbf{x}-\mathbf{y}) \cdot \mathbf{k}^{-1} \cdot(\mathbf{x}-\mathbf{y})-\frac{\left((\mathbf{x}-\mathbf{y}) \cdot \mathbf{k}^{-1} \cdot \mathbf{e}_{3}\right)^{2}}{\mathbf{e}_{3} \cdot \mathbf{k}^{-1} \cdot \mathbf{e}_{3}}=(\mathbf{x}-\mathbf{y}) \cdot \mathbf{k}_{2}^{-1} \cdot(\mathbf{x}-\mathbf{y})$.

By combining Eqs. (54), (55), and substituting the result in Eq. (52), we get:

$$
\begin{aligned}
G_{2}^{*}\left(\mathbf{T}^{*}(\mathbf{x}), \mathbf{T}^{*}(\mathbf{y})\right) & =\frac{1}{2 \pi k} \ln \left(\frac{\lambda^{\prime}}{\sqrt{k(\mathbf{x}-\mathbf{y}) \cdot \mathbf{k}_{2}^{-1} \cdot(\mathbf{x}-\mathbf{y})}}\right) \\
& =\frac{1}{2 \pi k} \ln \left(\sqrt{\frac{\left|\mathbf{k}_{2}\right|^{1 / 2}}{k}} \frac{\lambda^{\prime}}{\sqrt{\left|\mathbf{k}_{2}\right|^{1 / 4}(\mathbf{x}-\mathbf{y}) \cdot \mathbf{k}_{2}^{-1} \cdot(\mathbf{x}-\mathbf{y})}}\right)
\end{aligned}
$$

Finally, the relation between Green's functions in original and transformed domains is:

$$
G_{2}^{*}\left(\mathbf{T}^{*}(\mathbf{x}), \mathbf{T}^{*}(\mathbf{y})\right)=\alpha G_{2}(\mathbf{x}, \mathbf{y}),
$$

where $\alpha$ is given by Eq. (48) and $G_{2}$ is the anisotropic Green's function in $P$ computed with:

$\lambda=\lambda^{\prime} \sqrt{\frac{\left|\mathbf{k}_{2}\right|^{1 / 2}}{k}}$.

Combining this result with the transformation of the contour element $\mathrm{d} s$ shows that the term $q G_{2} \mathrm{~d} s$ in the BIE (Eq. (46)) is transformed into:

$G_{2}^{*} q^{*} \mathrm{~d} s^{*}$

and similarly for the term $u . q\left(G_{2}\right) \mathrm{d} s$. This proves that the transform of the BIE in $\mathbf{P}$ is the BIE in $\Pi$ written with the classical Green's function $G_{2}^{*}$ for isotropic problems.

\subsection{Expression of the characteristic lengths within $2 D$ Green's} tensors

It has been shown in [3] in the case of isotropic conductivity that the solution of the plane problem around a long cylinder approximates at best the 3D solution near the cylinder if $\lambda^{\prime}=2 a^{\prime}, 2 a^{\prime}$ being the length of the cylinder. As stressed before, the cylinder in the transformed domain is not generally a right cylinder and the assumption of "right" long cylinder at the basis of the result of [3] is not strictly fulfilled. However, it will be assumed in the following that the result obtained in [3] can be extended to oblique cylinders. Indeed, looking at the physical case of electrostatics, for cylinders that are long enough, the reason for which the characteristic length is equal to the length of the cylinder is the following: the end effects do not affect the distribution of the superficial density of sources around the mid plane of the cylinder that is characterized mainly by its length and its section. This physical consideration can be also applied to the case of an oblique cylinder.

Using this result, it is now possible to find the value of the characteristic length in the original space. From the relation between

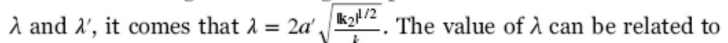
the length $2 a$ of the cylinder in the original domain by noticing from subSection 5.2.2 that the unit vector along the original cylinder is transformed into a vector of length $\alpha$ and therefore the lengths of the cylinders are related by $2 a^{\prime}=2 a \alpha$, leading to $\lambda=2 a \alpha \sqrt{\frac{\left.\mathbf{k}_{2}\right|^{1 / 2}}{k}}$. Replacing $\alpha$ by $\alpha=\sqrt{k \mathbf{e}_{3} \cdot \mathbf{K}^{-1} \cdot \mathbf{e}_{3}}$ leads to $\lambda=2 a \sqrt{\mathbf{e}_{3} \cdot \mathbf{K}^{1} \cdot \mathbf{e}_{3}}\left|\mathbf{k}_{2}\right|^{1 / 4}$.

It is exactly the expression of the field generated by a line source of length $2 a$. It shows that the Green's function leading to the solution of the plane problem that approximates at best the solution near the midplane corresponds to a line source whose length is the one of the cylinder. It generalizes the result obtained in the isotropic case [3].

\section{Numerical examples}

\subsection{Determination of the degenerate scale}

As an example of determination of degenerate scale, let us consider the case of an anisotropic operator with $\lambda=1$ and of an elliptic boundary $\Gamma$ with semi axes parallel to the coordinate axes with values $a_{1}$ and $a_{2}$. We assume also that the eigenvectors of $\mathbf{k}_{2}$ are also parallel to the coordinate axes.

The transformed boundary becomes an ellipse with semi axes $a_{1}\left|\mathbf{k}_{2}\right|^{1 / 4} / \sqrt{k_{1}}$ and $a_{2}\left|\mathbf{k}_{2}\right|^{1 / 4} / \sqrt{k_{2}}$. The degenerate scale in the case of the Laplace equation for the ellipse with semi-axes $a_{1}$ and $a_{2}$ being $2 /\left(a_{1}+a_{2}\right)$, the critical scale for $\Gamma$ and the anisotropic conduction equation is equal to:

$\rho_{0}=\frac{2}{\left(k_{1} k_{2}\right)^{1 / 4}\left(a_{1} / \sqrt{k_{1}}+a_{2} / \sqrt{k_{2}}\right)}$

If the axes of the ellipse are different from the principal axes of $\mathbf{k}$, we can consider the matrix of $\mathbf{T}$ in the basis with axes parallel to the semi axes of the ellipse and we can get the following result:

$$
\rho_{0}=\frac{2}{\sqrt{\left(a_{1} T_{11}+a_{2} T_{22}\right)^{2}+\left(a_{1}-a_{2}\right)^{2} T_{12}^{2}}} .
$$

In each case, the elliptic cylinder with the anisotropic operator and the elliptic boundary with semi-axes $a_{1} \rho_{0}$ and $a_{2} \rho_{0}$ is at the degenerate scale.

A numerical application has been effected for a cylinder with an elliptic cross section $a_{1}=1, a_{2}=2$ in an anisotropic medium with $k_{1}=3, k_{2}=1$.

The numerical representation of the integral equation by the Boundary Element Method using constant elements can be written as $[H][u]=[G][q]$

where $u$ contains the values of the potential at nodes of the boundary and $q$ contains the normal derivatives at nodes of the boundary. The degenerate scale corresponds to a null eigenvalue of $[G]$ matrix and therefore to a bad conditioning of this matrix around the degenerate scale.

As an example, Fig. 4 displays the condition number of matrix $[G]$

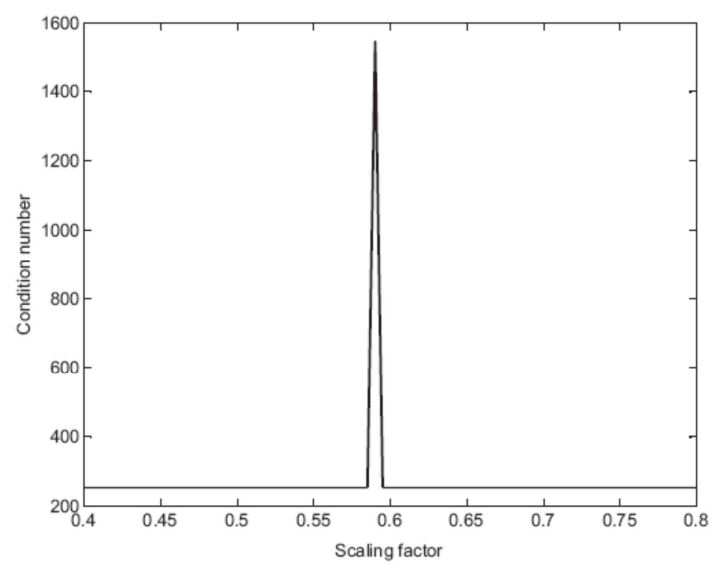

Fig. 4. Condition number of the matrix $[G]$ as a function of the scaling factor for a cylinder with an elliptic section in an anisotropic medium. 


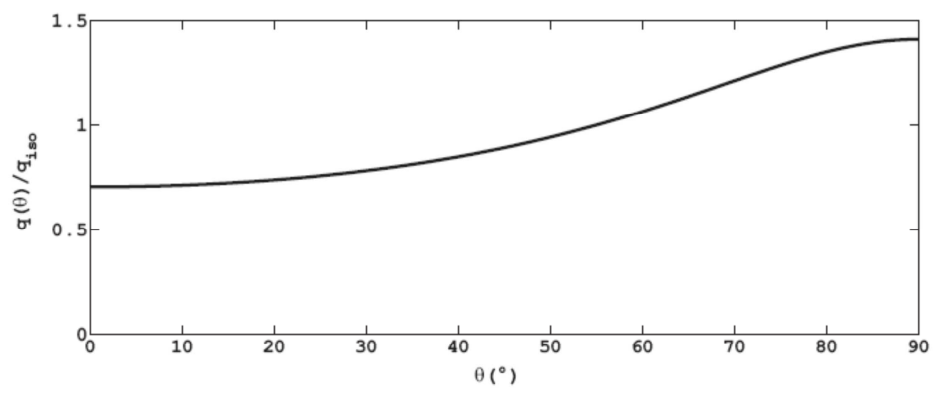

Fig. 5. Angular variation of the normal flux along the elliptic boundary.

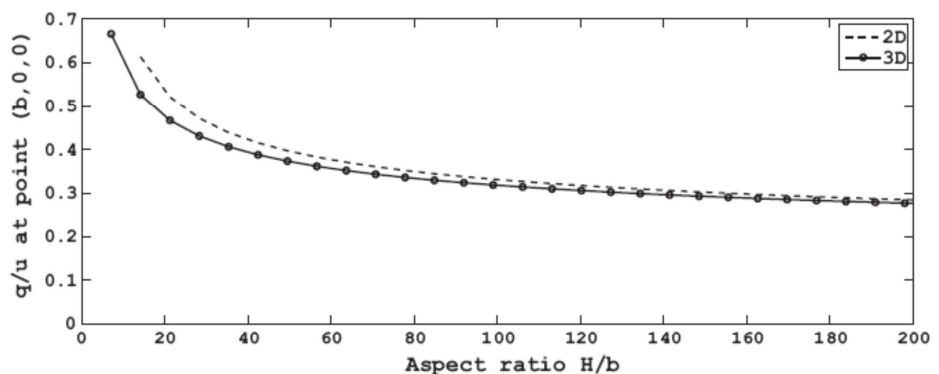

Fig. 6. Comparison between 2D and 3D values of the ratio $q / u$ at point $(b, 0,0)$ for different aspect ratios.

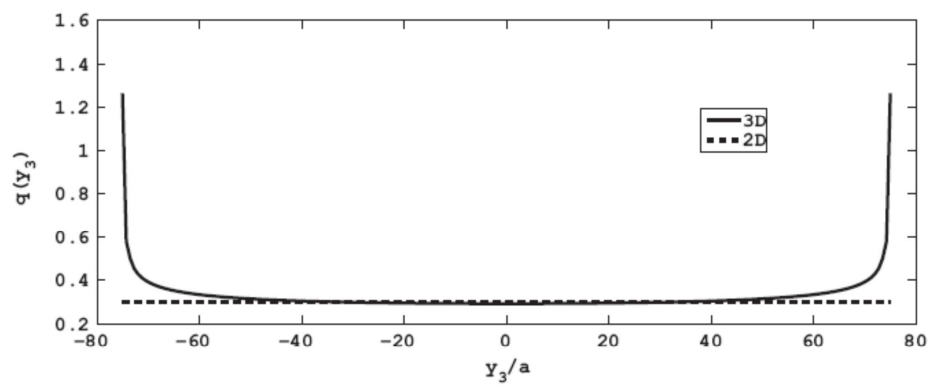

Fig. 7. Comparison between 2D and 3D values of the ratio $q / u$ at points $\left(b, y_{3}, 0\right)$ for an aspect ratio $H / b=150$.

as a function of the scaling factor for a matrix $[G]$ built by using 200 constants elements for the ellipse and material corresponding to the numerical application. The results display clearly an increase of the condition number of matrix $[G]$ around the scaling factor of 0.59 corresponding to the degenerate scale.

\subsection{Comparison of $3 D$ and $2 D$ solutions}

As an example, let us consider the case of the outer problem related to a right cylinder having an elliptic cross-section with radii $b$ along $y_{1}$ and $2 b$ along $y_{2}$. Its height $H=2 a$ is large compared with $b$ and the origin is at the center of the cylinder. The conductivity tensor is anisotropic with its principal directions along $y_{1}, y_{2}$ and $y_{3}$, with eigenvalues given by $k_{1}=1, k_{2}=4, k_{3}=2$. The matrix of the transformation $\mathbf{T}$ is diagonal with $T_{11}=\sqrt{2}, T_{22}=\sqrt{2} / 2, T_{33}=1$. Using $\mathbf{T}$, the cylinder is transformed into a circular cylinder with a radius $R=b . \sqrt{2}$ and the isotropic conductivity is $k=2$.

Let us consider the 3D Dirichlet problem related to a constant potential $u_{0}$ at the surface of the elliptic cylinder. This problem is transformed into a Dirichlet problem with a constant potential for a circular cylinder. This has been studied by numerous authors, as reported in [3]. Due to the axial symmetry for the circular problem under consideration, the potential is only a function $u=F\left(r, y_{3}^{\prime}\right)$ with $r=\sqrt{y^{\prime} 2_{1}+y^{\prime} 2_{2}}$. As a consequence, the solution in the plane $P$ outside the elliptic cylinder becomes $u=F\left(\sqrt{2 y_{1}^{2}+y_{2}^{2} / 2}\right)=F(\rho)$.

The coordinates of the points along the elliptic section can be parametrized by $y_{1}=\cos (\theta), y_{2}=2 \sin (\theta)$. Using the results of the subSection 5.2 .3 provides the relation between the boundary normal flux $q$ in the original problem and the one, $q^{*}$, in the transformed problem. This relation is given by

$$
\frac{q^{*}}{q}=\sqrt{\frac{\sin ^{2} \theta+4 \cos ^{2} \theta}{2}}
$$

Fig. 5 displays the angular distribution of the normal flux along the elliptic section that comes from this relation.

Due to the symmetry, the 3D solution in the isotropic case can be obtained by using a plane BEM solution in a vertical plane or approximate closed-form solutions. From another point of view, the 


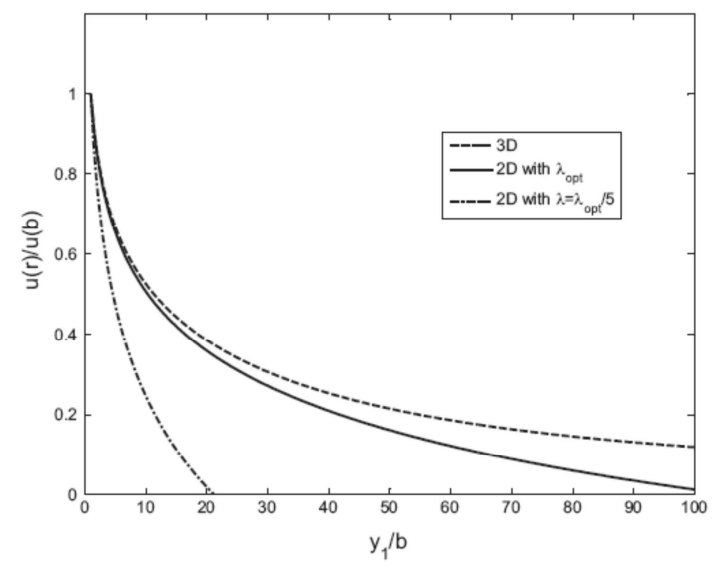

Fig. 8. Comparison between 2D and 3D values of $u\left(y_{1}, 0,0\right)$ for an aspect ratio $H / b=150$. The computation with $H$ corresponds to the optimized value of $\lambda$.

2D solution is given by:

$u=u_{0} \frac{\ln (\rho / H)}{\ln (b \sqrt{2} / H)}$

Fig. 6 displays the values of the ratio $q\left(y_{1}=b, y_{2}=0, y_{3}=0\right) / u_{0}$ for different values of the shape ratio $b / H$, showing that the 2D solution becomes accurate for values of $b / H$ around 100; the ratio depends on $\lambda$ as evaluated in subSection 5.3 .

Fig. 7 displays the value of the normal flux along the line $y_{1}=b$, $y_{2}=0$, for a shape ratio $H / b=150$ showing that the $2 \mathrm{D}$ solution is accurate in the vicinity of the cylinder for the domain between the horizontal planes $y_{3}=-40$ and $y_{3}=40$.

Fig. 8 displays the values of potential along the axis $O y_{1}$ as a function of $y_{1}$. It shows that the 2D solution with an optimized value of $H$ is a satisfying approximation of the 3D solution in the vicinity of the boundary, upto $y_{1} / b=10$. However, for large values of $y_{1}$, the 2D solution becomes inaccurate. More specifically, it can be seen that the 2D solution becomes null for $y_{1} / b=100$, that is not physical, as show the 3D solution. For comparison, another 2D result is reported for a value of the constant computed with $H / 5$ and which is not optimized. This last result shows a larger difference between this $2 D$ solution and the 3D solution, even in the vicinity of the cylinder, than for the optimized $2 D$ solution.

This example shows that the $2 \mathrm{D}$ solution with a convenient choice of the 2D Green's tensor approximates correctly the 3D solution in the vicinity of the median plane and of the inner boundary, as it has been predicted in the previous section. This is clearly of interest for numerous engineering applications.

For most of general examples, the 3D solution has no more circular symmetry in the isotropic transformed problem. Using BEM is still possible, but with large aspect ratios of the cylinder, the boundary surface would need a large quantity of surface elements, to respect usual limits on the shape of boundary elements. In comparison, the 2D solution uses only a simple discretization of the elliptic boundary and it shows clearly the interest of the results developped in this paper.

\section{Conclusion}

The fields generated by segment sources related to anisotropic conduction have been computed for any orientation with respect to the eigenvectors of the conductivity tensor, recovering the classical 2D
Green's function for anisotropic conduction with a characteristic lengthscale.

Next, following classical considerations, a linear point transformation has been introduced, that produces an associated problem characterized by isotropic conduction. The boundary conditions related to this problem with isotropic conduction have been obtained from the boundary conditions on the original problem. The relation between Green's functions related to the original plane problem and to the associated one have been provided.

The linear transformation has been used to show the relation between degenerate scales in the original domain and in the transformed domain. Finally, the solutions of exterior BVP with cylindrical boundaries obtained by BIE have been considered. It has been shown that, as for the case of isotropic conduction, the best lengthscale to introduce into the 2D Green's function corresponds to a segment source whose length is equal to the height of the cylindrical domain.

\section{References}

[1] Jaswon MA. Integral equation methods in potential theory, I. Proc R Soc Lond 1963;A275:23-32.

[2] Bonnet M. Boundary integral equations methods for solids and fluids. N.Y: Wiley?

[3] Bonnet G, Corfdir A, Nguyen M-T. On the solution of exterior plane problems by the boundary element method: a physical point of view. Eng Anal Bound Elem 2014:38:40-8.

[4] Chadwick E, Hatam A. Slender body expansions in potential theory along a finite straight line. Z fur Angew Math Phys 2010;61:493-508.

[5] Rafiezadeh K, Ataie-Ashtiani B. Seepage analysis in multi-domain general anisotropic media by three-dimensional boundary elements. Eng Anal Bound Elem 2013;37:527-41.

[6] Eskola L. Reflections on the electrostatic characteristics of direct current in an anisotropic medium. Geoexploration 1988:25:211-7.

[7] Hänninen JJ, Nikoskinen KI, Lindell IV. Electrostatic image theory for two anisotropic half-spaces. Electr Eng 2005;88:1-12.

[8] Carslaw HS, Jaeger J. Conduction of heat in solids, 2nd ed.. Oxford: Clarendon Press; 1959.

[9] Onsager L. Reciprocal relations in irreversible processes. I. Phys Rev 1931;37:405-26.

[10] Onsager L Reciprocal relations in irreversible processes. II. Phys Rev $1931 ; 38: 2265-79$

[11] Powers JM. On the necessity of positive semi-definite conductivity and Onsager reciprocity in modeling heat conduction in anisotropic media. Trans ASME 2004; 126:670-5

[12] Hahn DW, Özişik MN. Heat conduction, 3rd ed. Hoboken, New Jersey: Wiley; 2012.

[13] Mantič V, París F, Berger J. Computational Mechanics '95: theory and applications. In: Atluri SN, Yagawa G, Cruse T, editors. . On free terms and singular integrals in isotropic and anisotropic potential theory, 2 1995; 1995. p. 2806-11.

[14] Marczak RJ, Denda M. New derivations of the fundamental solution for heat conduction problems in three-dimensional general anisotropic media. Int $\mathrm{J}$ Hea Mass Transf 2011;54:3605-12.

[15] Godement R. Algebra. Paris: Hermann; 1968.

[16] Chang Y-P, Kang CS, Chen DJ. The use of fundamental Green's functions for the solution of problems of heat conduction in anisotropic media. Int J Heat Mass

[17] Chao BT. A note on conduction of heat in anisotropic media. Appl Sci Res

[18] Mulholland G, Gupta B. Heat transfer in a three-dimensional anisotropic solid of arbitrary shape. J Heat Transf 1977;99(1):115-28.

[19] Poon $\mathrm{K}$, Chang $\mathrm{Y}$. Trans or antion of heat conduction problems from anisotropic to isotropic. Lett Heat Mass Transf 1978;5:215-21.

[20] Poon K, Tsou P, Chang Y. Solution of anisotropic problems of first class by coordinate-transformation. J Heat Transf 1979;101(2):340-5.

[21] Shit Y, Tan CL. BEM treatment of two-dimensional anisotropic field problems by direct domain mapping. Eng Anal Bound Elem 1997;20:347-51.

[22] Shiah Y, Tan CL. BEM treatment of three-dimensional anisotropic field problems by direct domain mapping. Eng Anal Bound Elem 2004;28:43-52

[23] Mantic V, París F, Berger J. Singularities in 2D anisotropic potential problems in multi-material corners: Real variable approach. Int $J$ Solids Struct 2003;40(20):5197-218.

[24] Ciarlet PG. Linear and nonlinear functional analysis with applications. Philadelphia: SIAM: 2013.

[25] Rumely RS. Capacity Theory on Algebraic Curves. Berlin: Springer-Verlag; 1989. 